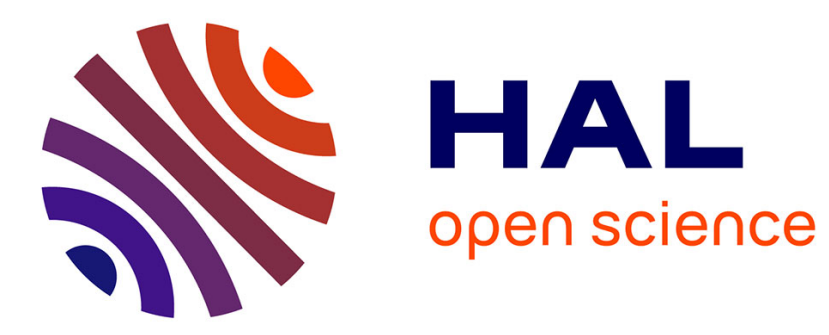

\title{
Normalized site attenuation standard correction from numerical computing
}

\author{
A. Askri, Christian Vollaire, Laurent Nicolas, Daniel Prebet
}

\section{To cite this version:}

A. Askri, Christian Vollaire, Laurent Nicolas, Daniel Prebet. Normalized site attenuation standard correction from numerical computing. IEEE Transactions on Magnetics, 2002, 38 (2 Part 1), pp.693696. hal-00140463

\section{HAL Id: hal-00140463 https://hal.science/hal-00140463}

Submitted on 18 Apr 2007

HAL is a multi-disciplinary open access archive for the deposit and dissemination of scientific research documents, whether they are published or not. The documents may come from teaching and research institutions in France or abroad, or from public or private research centers.
L'archive ouverte pluridisciplinaire HAL, est destinée au dépôt et à la diffusion de documents scientifiques de niveau recherche, publiés ou non, émanant des établissements d'enseignement et de recherche français ou étrangers, des laboratoires publics ou privés. 


\title{
Normalized Site Attenuation Standard Correction From Numerical Computing
}

\author{
A. Askri, C. Vollaire, L. Nicolas, and D. Prebet
}

\begin{abstract}
In this paper, a numerical model for site attenuation is described. Both horizontal and vertical polarization are presented. Ideal site attenuation values are compared with three-dimensional (3-D) finite-element results in the frequency range required by the standards. The results show significant errors in computing 3-meter $(\mathrm{m})$ site attenuation in the 30-300 $\mathrm{MHz}$ frequency range. New values for the normalized site attenuation are then given for 3-m measurements. Such a numerical tool can be used to optimize the performances of indoor test site such as semi-anechoic chamber.
\end{abstract}

Index Terms-Antenna factor, semi-anechoic chamber, site attenuation, propagation.

\section{INTRODUCTION}

B ECAUSE it is difficult to have convenient space for open area test site (OATS), normalized site attenuation (NSA) has become the standard method for determining the acceptability of electromagnetic compatability test sites to perform radiated emission measurements. The criteria for site acceptance is specified in ANSI C63.4 as well as other standards [1]. It is based on a comparison of the measured NSA for a given site to the calculated theoretical NSA of an ideal site. The ANSI C63.4 Standard requires that the measured NSA must be within $\pm 4 \mathrm{db}$ of the theoretical NSA in the frequency range of 30-1000 MHz in order the site under evaluation to be considered acceptable for performing radiated emission testing [1].

Semi-anechoic chambers are currently used for indoor antenna measurements, electromagnetic interference measurements and electromagnetic compatibility measurements [2]. For such systems, the back reflections due to the walls are minimized in order the sources to radiate like in an OATS. Performances required for a test site are based on the ideal site attenuation model developed by Smith et al. [3].

Many researchers have taken delight of the exact theoretical relations that could be compared with practical measurements experience at their different centers of measurement, and their different models. All the presented results show a good agreement between measured and theoretical values. The maximum deviation is below the $\pm 4 \mathrm{db}$ specification required by ANSI 63.4. The method proposed by American National Standard Institute (ANSI) [1] and [3] for the evaluation of the NSA is obtained with a geometrical optics approximation [1], [3]. Practical experience has shown that correction factors must be added on the general formulation of NSA in order to take into account

Manuscript received February 21, 2001; revised October 25, 2001.

The authors are with the CEGELY, UMR CNRS 5005, 69131 Ecully Cedex, France (e-mail: laurent.nicolas@eea.ec-lyon.fr).

Publisher Item Identifier S 0018-9464(02)01227-X. several effects. In [4] and [5], the assumptions in the method introduced by the ANSI are analyzed. A new approach based on the self and mutual impedance between the antennas (tuned dipoles and broadband antennas) is examined. The results presented in [4] and [5] show that the mutual impedance is much lower than the sum of self-impedance and load impedance. Furthermore, the self-impedance is approximated by a simplified equations and the antennas factors do not change with the antenna height. In [6] a near-field correction factor is presented for the 30-Mhz to 200-MHz frequency range for 3- and 10-meters (m) distances and for both horizontal and vertical polarizations. Furthermore, the introduced modification of the equations for calculating NSA with the complete electric field shows that the largest errors occur for horizontal polarization at a frequency of $30 \mathrm{MHz}$. It is recommended the complete expression for the electric field of a point dipole should be used in calculating site attenuation and in calibrating antennas for measuring site attenuation. A correction for infinite conducting ground plane is also introduced.

In [7], the NSA can be similarly characterized on the ideal open-field test site by defining the effective distance from the source to the observation point. Numerical results for horizontal polarization agree with those of the ANSI. However, the results do not agree for vertical polarization. In [8], a new model based on a complex image theory is presented. It can affiliate nearfield correction factored the contribution of Norton surface wave component but only in the case of the vertical electric dipole.

In this paper we propose a method to compute the NSA numerically. This method is based on a three-dimensional (3-D) finite-element (FE) formulation. Numerical results are compared with analytical ones. New values of NSA are given for 3-m measurements. This numerical formulation is then used to predict the NSA of semi-anechoic chamber. This technique allows a low-cost design of semi-anechoic chambers.

\section{TheOREticAl Normalized Site AtTENUATion}

Site attenuation is measured by using a pair of tuned dipole antennas. The standard (ANSI Standard c63.4-1988) sets the procedures for the NSA measurements on a test site [1]: two antennas are set up on the test site using specified geometry as shown in Fig. 1. The NSA procedure requires two different measurements of received voltage $V r$. The first reading of $V r$ is made with two coaxial cables disconnected from the antennas and connected to each other via an adapter. The second reading of $V r$ is performed with the coaxial cables reconnected to their respective antennas, and the maximum signal is measured when the receiving antenna's height is scanned. The signal source 


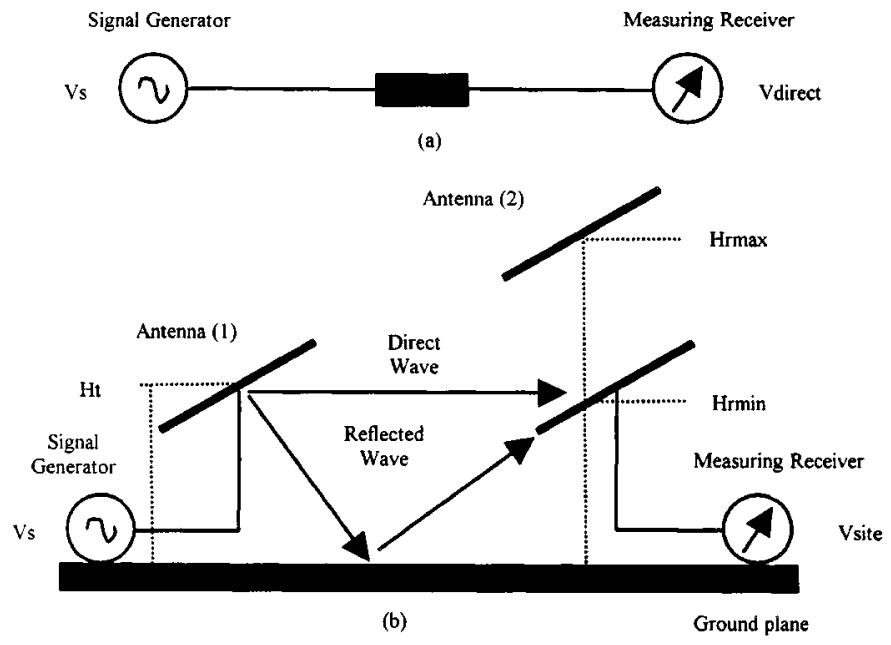

Fig. 1. Measurement receiving voltage. (a) $V$ direct and (b) $V$ site.

voltage $V s$ is kept constant. The first reading of $V r$ is called $V_{\text {DIRECT }}$ and the second is $V_{\text {SITE }}$. These values are used in the following equation for the measured NSA. All terms are in decibel [4]

$$
\mathrm{NSA}=V_{\text {DIRECT }}-V_{\mathrm{SITE}}-\mathrm{AF} 1-\mathrm{AF} 2-\Delta \mathrm{AF}_{\mathrm{TOT}}
$$

where

AF1 transmitting antenna factor;

$\mathrm{AF} 2$ receiving antenna factor;

$\Delta \mathrm{AF}_{\text {TOT }}$ mutual-impedance correction factor.

The ideal site attenuation is calculated from the model developed in [3]. The basic model for a semi-anechoic chamber is shown in Fig. 2. The total field in the receiving point is the superposition of the field in direct path and indirect path. A convenient choice of site attenuation could be expressed in terms of antennas factors of receiving and transmitting antennas, respectively, $\mathrm{AF}_{R}$ and $\mathrm{AF}_{T}$

$$
A=\frac{79.58 \mathrm{AF}_{R} \mathrm{AF}_{T} \sqrt{49.2}}{2 F_{m} E_{D}^{\max }} .
$$

The normalized site attenuation is then defined by dividing (2) by the $\mathrm{AF}_{S}$ of the two antennas

$$
A_{N}=\frac{A}{\mathrm{AF}_{R} \mathrm{AF}_{T}}=\frac{79.58 \sqrt{49.2}}{2 F_{m} E_{D}^{\max }} .
$$

Written in decibels, the NSA becomes

$$
A_{N}(\mathrm{~dB})=-20 \log F_{m}+48.92-E_{D}^{\max }(\mathrm{dB} . \mu \mathrm{V} / \mathrm{m})
$$

where $F_{m}$ is the frequency $(\mathrm{MHz})$ and $E_{D}^{\max }(\mathrm{dB} . \mu \mathrm{V} / \mathrm{m})$ is the maximum received electric field from a theoretical half-wave dipole with one picowatt of radiated power and receiving antenna located at $h_{2}^{\min } \leq h_{2} \leq h_{2}^{\max }$. The process is repeated for a receiving antenna located at heights varying between 1 and 4 $\mathrm{m}$ to obtain $E_{D}^{\max }(\mathrm{dB} . \mu \mathrm{V} / \mathrm{m})$ because the measured attenuation is affected by more than just the site and the antenna factors. Table I shows the lengthy algebraic equation required of calculations. Several different correction factors may be introduced if the antennas have changeable antenna factors [4].

Figs. 3 and 4 show the theoretical site attenuation for the maximum received electric field, for different frequencies and for both horizontal and vertical polarization. The formulation in [3] assumes that the receiving antenna is in the far field region

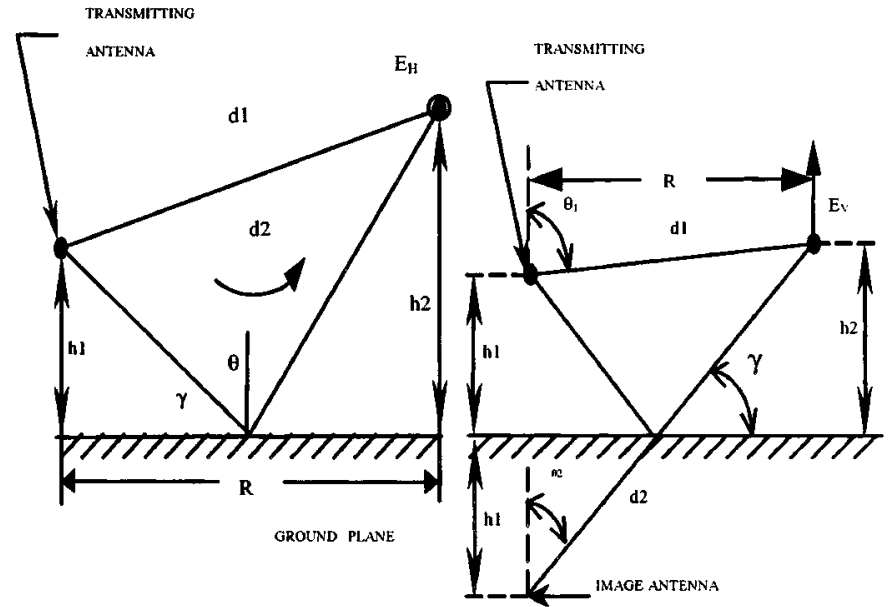

Fig. 2. Propagation geometry for horizontal (left) and vertical (right) polarization.

TABLE I

THEORETICAL MODEL FOR NSA

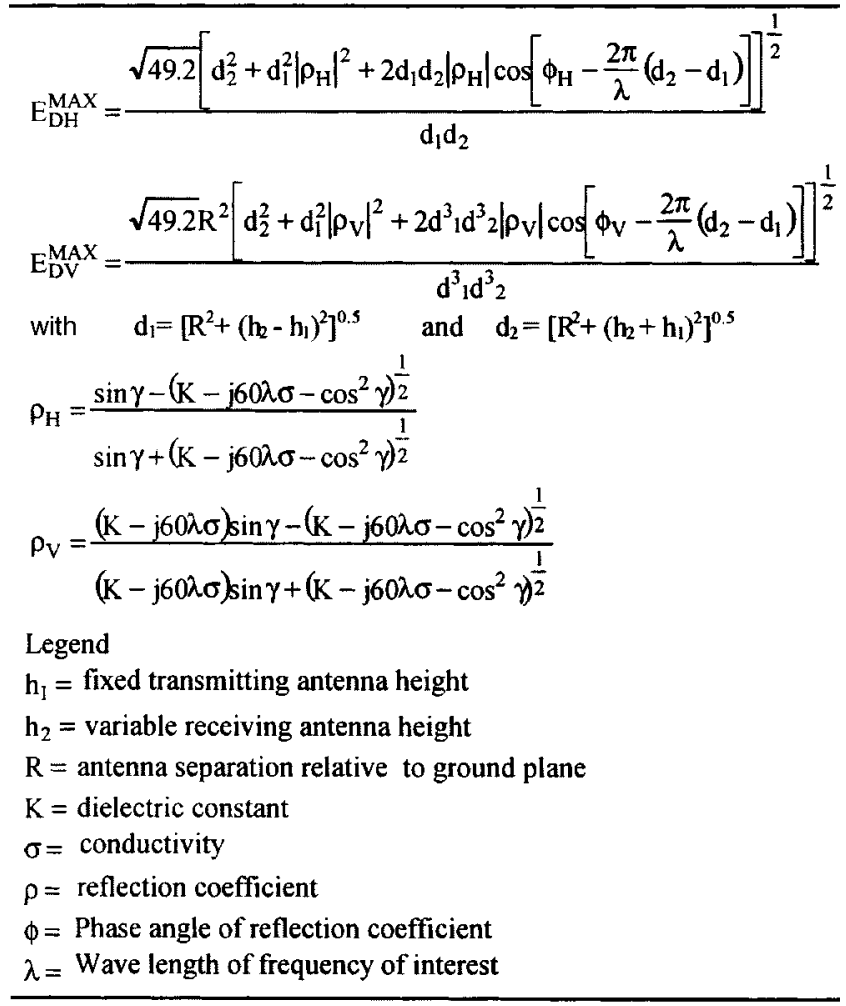

of the transmitting antenna. If not the case, the E-field falls off as an inverse function of $R$ (distance). Further study shows that theoretical results are innacurate, especially at lower frequencies. It is possible to get an error at $30 \mathrm{MHz}$ by disregarding $1 / R^{2}$ and higher terms [6].

\section{NUMERICALLY COMPUTED NSA}

The developed FE formulation works in the frequency domain [9]. It can handle dielectric, permeable or absorbing materials. Absorber losses (dielectric and finite conductivity) are combined in the complex permittivity. Free-space radiation is taken into account using absorbing boundary conditions. In the 


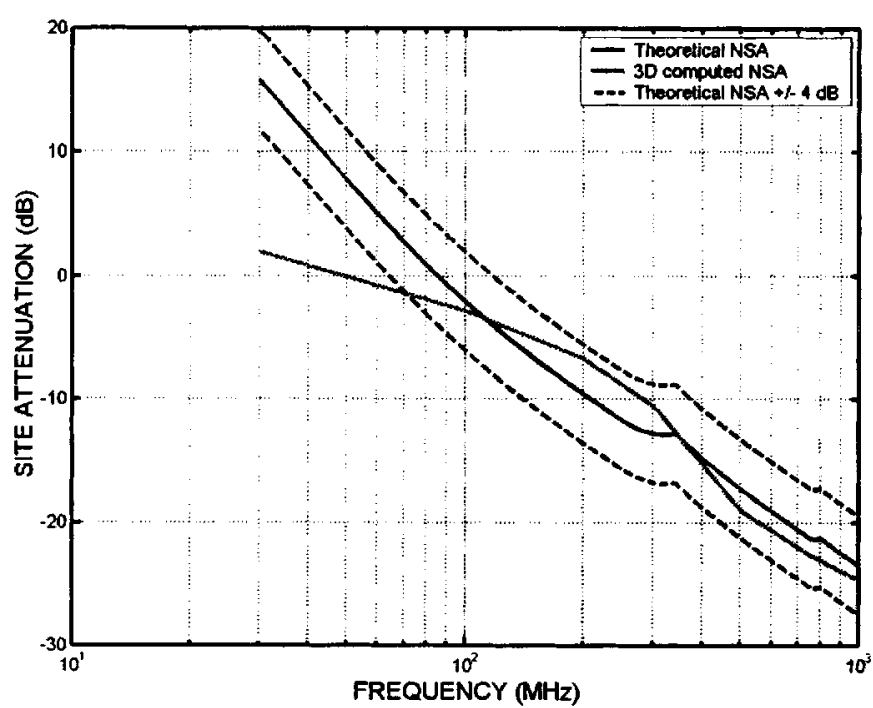

Fig. 3. Horizontal NSA (3 m).

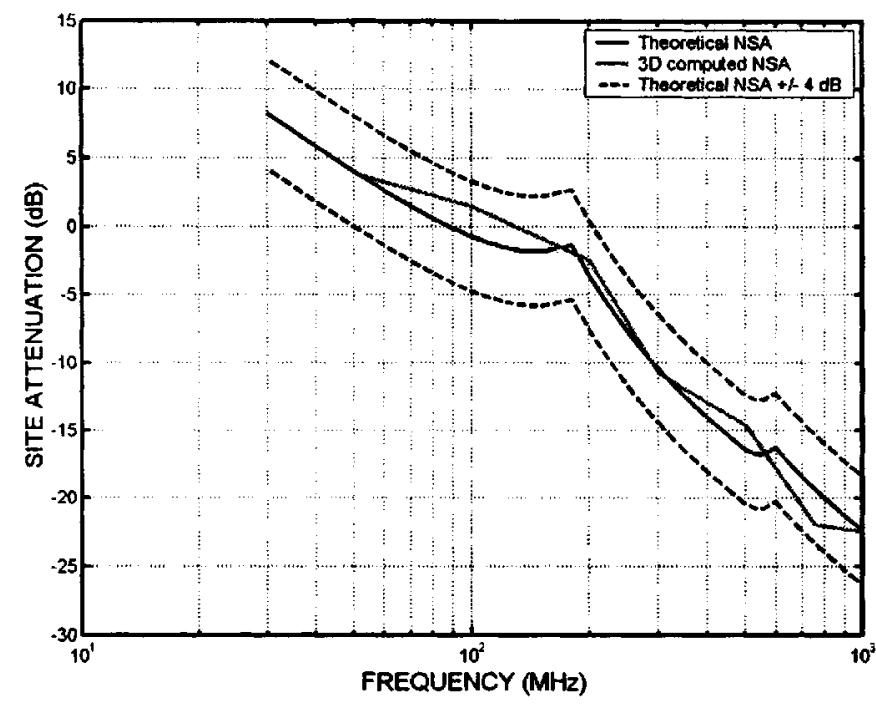

Fig. 4. Vertical NSA (3 m).

case of the Faraday box, the walls are modeled as perfect electric conductors.

This formulation is directly written in terms of $\mathbf{E}$ or $\mathbf{H}$ vector fields. Vector FE are used (first-order tetrahedral), leading to one complex unknowns per edge of the mesh. This allows to take into account different material interfaces such as air-absorbers or absorber-wall. To obtain a good accuracy, ten nodes per wavelength are at least necessary

$$
\begin{gathered}
\iiint_{v}\left(\frac{1}{\mu_{r}} \nabla \times E\right) \cdot(\nabla \times W) d v-k_{0}^{2} \iiint_{v}\left(\varepsilon_{r} E . W\right) d v \\
+\oint_{\Gamma} \frac{1}{\mu_{r}} T(E) . W d \Gamma=-j \omega \mu_{0} \iint_{s} J e . W d S
\end{gathered}
$$

where

Je source term;

$T$ three-dimensional Engquist Majda ABC;

$\Gamma \quad$ external surfaces;

$S$ source surface.

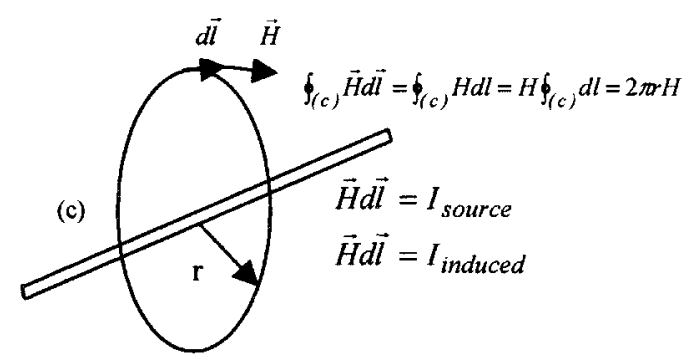

Fig. 5. Application of Ampere's law.

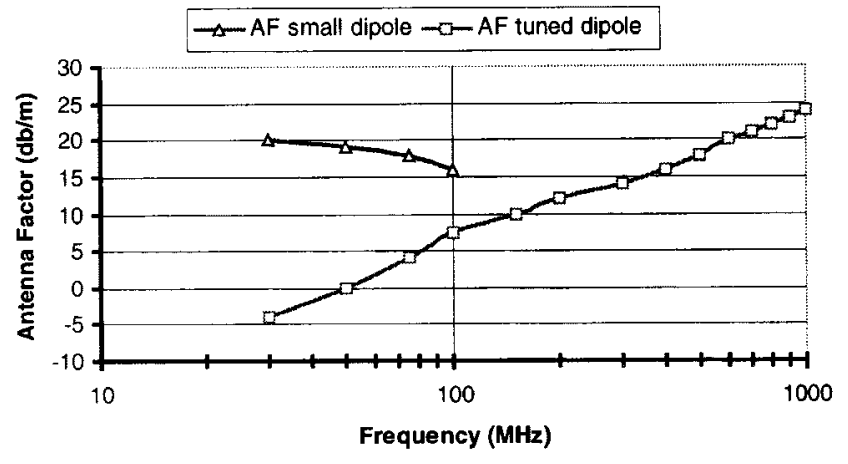

Fig. 6. Antenna factors for tuned and small dipoles.

The transmitting antenna is modeled with an initial sinusoidal current distribution for tuned dipoles and with a triangular current distribution for small dipoles. Thin wire element has been introduced in the geometry to simulate the receiving antennas. It has been shown that the back scattering effect on the transmitting antennas can be neglected. NSA is computed from the rate of both currents on transmitting antenna and on receiving antenna obtained by using the Ampere's law around the antennas (Fig. 5).

In the numerical formulation, the current density is imposed on the transmitting antenna. In the $200-\mathrm{MHz}$ to $1-\mathrm{GHz}$ frequency range, accorded dipole antennas are used as transmitting and receiving antennas. At frequencies below $200 \mathrm{MHz}$, the NSA is evaluated with dipole accorded to $200 \mathrm{MHz}$ (especially for vertical polarization). For the vertical polarization, the far end of the antenna should be kept at $25 \mathrm{~cm}$ or more above the metal ground plane. The computed NSA is obtained by applying (6)

$$
\mathrm{NSA}=\mathrm{SA}-2 \mathrm{AF} \text {. }
$$

SA is the site attenuation between transmitting and receiving antennas, and $\mathrm{AF}$ is the factors of the transmitting and receiving antennas. In terms of current NSA is given by

$$
\mathrm{NSA}(\mathrm{db})=20 \log \frac{I_{\text {source }}}{I_{\text {induced }}}-2 \mathrm{AF} \text {. }
$$

The AF of transmitting and receiving antennas is calculated by using a time-domain integral formulation based on antenna theory [10]. AF is defined by using the ratio of electric field of incident electromagnetic wave arriving at an antenna $(1 \mathrm{~V} / \mathrm{m})$ to the voltage induced by the incident wave on the antenna. For tuned dipoles, the induced current is multiplied by the radiation resistance to obtain the induced voltage $[Z=R r$ in (8)]. For 


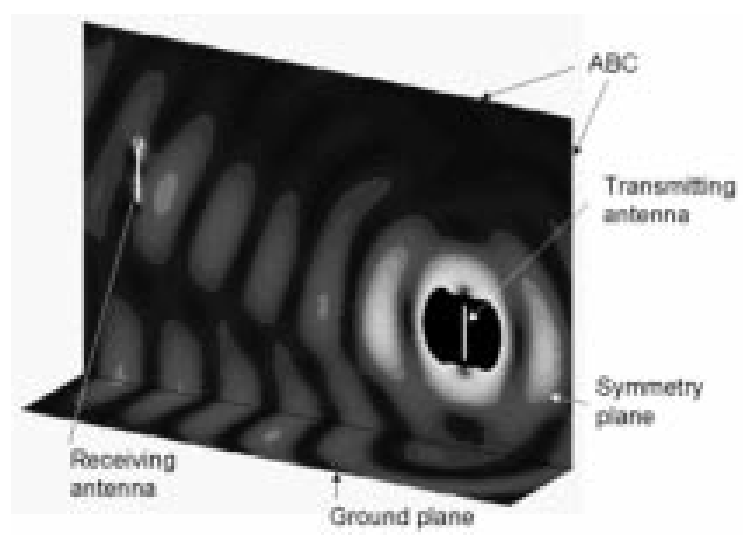

Fig. 7. Three-dimensional computation of NSA (instantaneous E field).

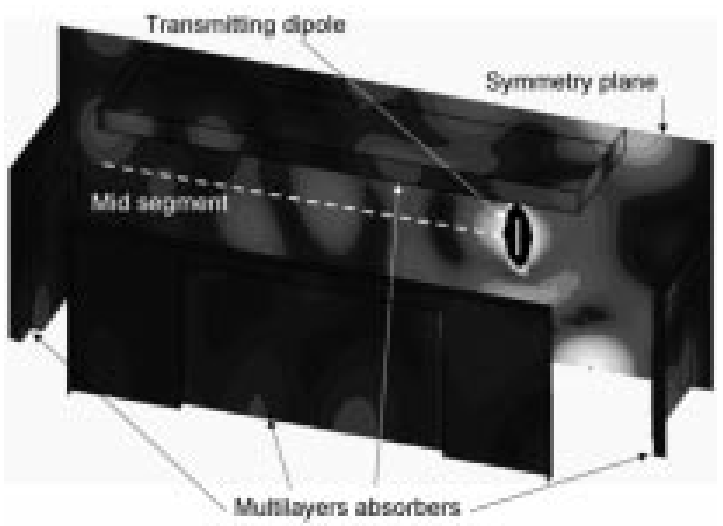

Fig. 8. E field on symmetry plane and on dielectric absorbing material in the anechoïc chamber (vertical polarization-200 MHz).

small dipoles used in vertical polarization below $200 \mathrm{MHz}$, the induced current is multiplied by the modulus of the complex impedance of the small dipole [11]. So AF is given by

$$
\mathrm{AF}(\mathrm{dB})=20 \log \frac{1}{\frac{I_{\text {induced }}}{\sqrt{2}}|Z|} .
$$

Fig. 6 shows the results of AF calculations using a TimeDomain Integral Formulation in the case of small dipole and tuned dipole [10], [12].

Fig. 7 shows the instantaneous E field for a 3-D simulation for vertical polarization $(f=750 \mathrm{MHz})$ and in free space with ground plane. Computation has been performed on a HP station J5000 (PA risk 8500 processor with 1 Go of RAM). The solver is the CG with SSOR preconditioning. The geometry is meshed with 56780 nodes and 304189 first-order tetrahedral elements leading to $10 \mathrm{~h}$ of CPU time.

From Figs. 3 and 4, it is shown that the theoretical NSA is accurately calculated for vertical polarization. In horizontal polarization, at low frequencies, large differences may be observed between theoretical and numerical results. The assumptions made in the theoretical model - antennas are modeled as point sources and far field approximation—are not valid even if

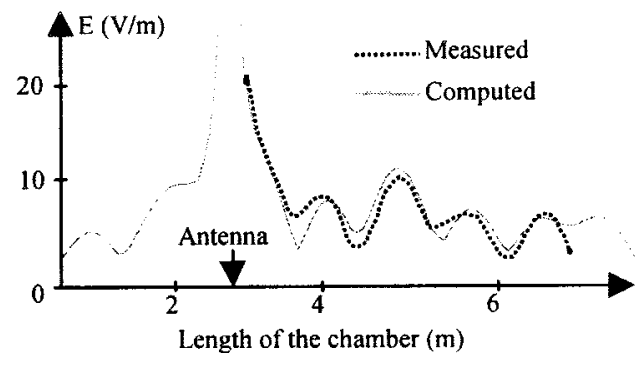

Fig. 9. E field on a mid segment (vertical polarization-200 MHz).

wide-band antennas such as log periodic or biconical antennas are used.

\section{Simulation of Semi-Nechoic Chamber}

An example of 3-D simulation of stationary waves in an semianechoic chamber with a dipole as transmitting antenna for vertical polarization is shown in Fig. 8. Dimensions of the chamber are $7.5 \times 4.5 \times 3 \mathrm{~m}$. Absorption is obtained using multilayer composite absorbers. In the anechoïc chamber, the validation has been performed for both vertical and horizontal polarization and for several frequencies by comparing computed field and measured field with small probes (Fig. 9).

The NSA of the semi-anechoïc chamber can be computed for a receiving antenna located at different heights and results can be compared with the theoretical values. New configurations of absorbers can be easily tested numerically.

\section{REFERENCES}

[1] Method of measurement of radio-noise emissions from low-voltage electrical and electronic equipment in the range of $10 \mathrm{kHz}$ to $1 \mathrm{GHz}$, ANSI Standard C63.4, 1988.

[2] “ C. I. S. P. R. 16 Specification for Radio Interference Measuring Apparatus and Measurement Methods," International Electromechanical Commission.

[3] A. A. Smith, Jr., R. F. German, and J. B. Pate, "Calculation of site attenuation from antenna factors," IEEE Trans. Electromagn. Compat., vol. EMC-24, pp. 301-316, Aug. 1982.

[4] A. Sugiura, "Formulation of normalized site attenuation in terms of antenna impedances," IEEE Trans. Electromagn. Compat., vol. 32, pp. 257-263, Nov. 1990.

[5] A. Sigura, T. Shinozuka, and A. Nishikata, "Correction factors for normalized site attenuation," IEEE Trans. Electromagn. Compat., vol. 34, pp. 461-470, Nov. 1992.

[6] J. D. Gavenda, "Near-field corrections to site attenuation," IEEE Trans. Electromagn. Compat., vol. 36, pp. 213-220, Aug. 1994.

[7] W. S. Bennett, "Normalized site attenuation newly characterized," in Proc. 1998 IEEE Int. Symp. (Electromagnetic Compatibility, 1998), pp. 141-146.

[8] P. T. Trakadas and C. N. Capsalis, "A mixed model for determination of normalized site attenuation in OATS," IEEE Trans. Electromagn. Compat., vol. 43, pp. 29-36, Feb. 2001.

[9] J. L. Yao-Bi, L. Nicolas, and A. Nicolas, " $H$ (curl) elements on hexahedral and vector A.B.C.'s for unbounded microwave problems," IEEE Trans. Magn., vol. 31, pp. 1538-1541, May 1995.

[10] F. Bost, G. Rojat, and L. Nicolas, "A time-domain integral formulation for the scattering by thin wires," IEEE Trans. Magn., vol. 36, pp. 868-871, July 1999.

[11] C. A. Balanis, Antenna Theory. New York: Wiley, 1982.

[12] Y. Takiguichi, E. Kimura, and M. Takuda, "NSA characteristics of anechoïc chamber using FDTD method," in Proc. 4th Eur. Symp. Electromagnetic Compatability (EMC Europe 2000 Brugge), pp. 257-262. 